\title{
Microfluidic engineering for continuous in-flow cyto-tomography
}

\author{
Pasquale Memmolo ${ }^{1, *}$, Massimiliano M. Villone ${ }^{2}$, Francesco Merola ${ }^{1}$, Lisa Miccio ${ }^{1}$, Martina Mugnano ${ }^{1}$, Pier Luca \\ Maffettone $^{2}$, and Pietro Ferraro ${ }^{1}$ \\ ${ }^{1}$ National Research Council of Italy, Institute of Applied Sciences and Intelligent Systems “E. Caianiello”, Via Campi Flegrei 34, \\ Pozzuoli, Naples, Italy. \\ ${ }^{2}$ Dipartimento di Ingegneria Chimica, dei Materiali e della Produzione Industriale, University of Naples "Federico II", Piazzale Tecchio \\ 80, 80125 Napoli, Italy.
}

\begin{abstract}
The possibility to investigate cells in microfluidic flow by using a full 3D morphometry analysis is highly demanded to achieve information about their healthiness. Recently, the tomographic flow cytometry by digital holography has been demonstrated to monitor red blood cells in microfluidics environment by simply applying flux pressure to induce random self-rotation of flowing cells. Here, we provide a microfluidic solution to engineer the flow with the aim to ensure the full 360 degree of angle rotation of all cells in the field of view. We test the proposed methods for circulating tumour cells.
\end{abstract}

\section{Introduction}

Quantitative phase imaging by digital holography is one of the most powerful imaging solution to investigate living biological samples [1-3]. The quantitative phasecontrast images, that can be recovered by holographic imaging in microscope configuration, encode the combination of two biophysical information of imaged cells, i.e. their refractive index and physical thickness. Therefore, it is possible to measure them only after a suitable decoupling procedure $[4,5]$. On the other hand, tomographic phase microscopy (TPM) [6,7] permits to retrieve the 3D refractive index mapping of cells, when a multiple observation from different views is implemented. Actually, three main approaches are known to image cells from different directions, i.e. beam deflaction [8], mechanical rotation [9] and microfluidic induced rotation $[10,11]$.

TPM by beam deflection suffers from some limitations, mainly related to the limited angle scanning range (up to 180 degrees), while TPM by mechanical rotation is intrinsically invasive, due to the need of manipulating cells. Actually, a different solution that uses an external force to manipulate cells have been proposed in ref. [12], where optical tweezers are used to trap and rotate cells. Of course, this method needs to a more complicate optical arrangement related to the use of a second light source to implemented the optical trapping [13].

Recently, the Tomographic flow cytometry (TFC) has been demonstrated [10], which overcome the limitation of the angle scanning range, providing the full 360 degrees of cells rotation, along with standard optical set-up. In fact, it uses digital holography in microscopy as the imaging tool and a microfluidic channel in which cells move in flow. TFC is successfully applied to reconstruct the tomogram of red blood cells and diatoms. However, the induced rotation of cell is not controlled, thus limiting the throughput of the proposed tomography system. Here we show a different solution to overcome this problem, by a microfluidic engineering of the flow through the fluid dynamic modelling. In particular, we design the physical sizes of the microfluidic channel along with the optimal condition of the pressure pump to ensure the cells tumbling, i.e. the rotation of cells without mechanical deformation [11]. In this case, the proposed TFC is suitable for the continuous in-flow cyto-tomography.

\section{Results and discussion}

The TFC by digital holography apparatus consist of a holographic microscope which makes imaging of flowing cells in a microfluidic channel. The cells tumbling is induced by the external force, through a controlled pressure pump. The cells moves and rotate continuously in the imaged field of view and the holographic recording with high frame rate is performed. After recording of video sequence of digital holograms, the numerical processing is implemented to recover the quantitative phase-contrast images. In particular, the automatic focusing algorithm is used to retrieve the axial position of each cells in the field of view, then the holographic 3D tracking [14] is employed to realign the detected cells and a suitable algorithm is used to calculate the tumbling angles frame-by-frame $[10,11]$. Finally, the tomogram is obtained by using the optical projection tomography method. In figure 1 , the complete pipeline of the TFC is reported with an example of application in the case of circulating breast cancer cells.

\footnotetext{
* Corresponding author: p.memmolo@isasi.cnr.it
} 


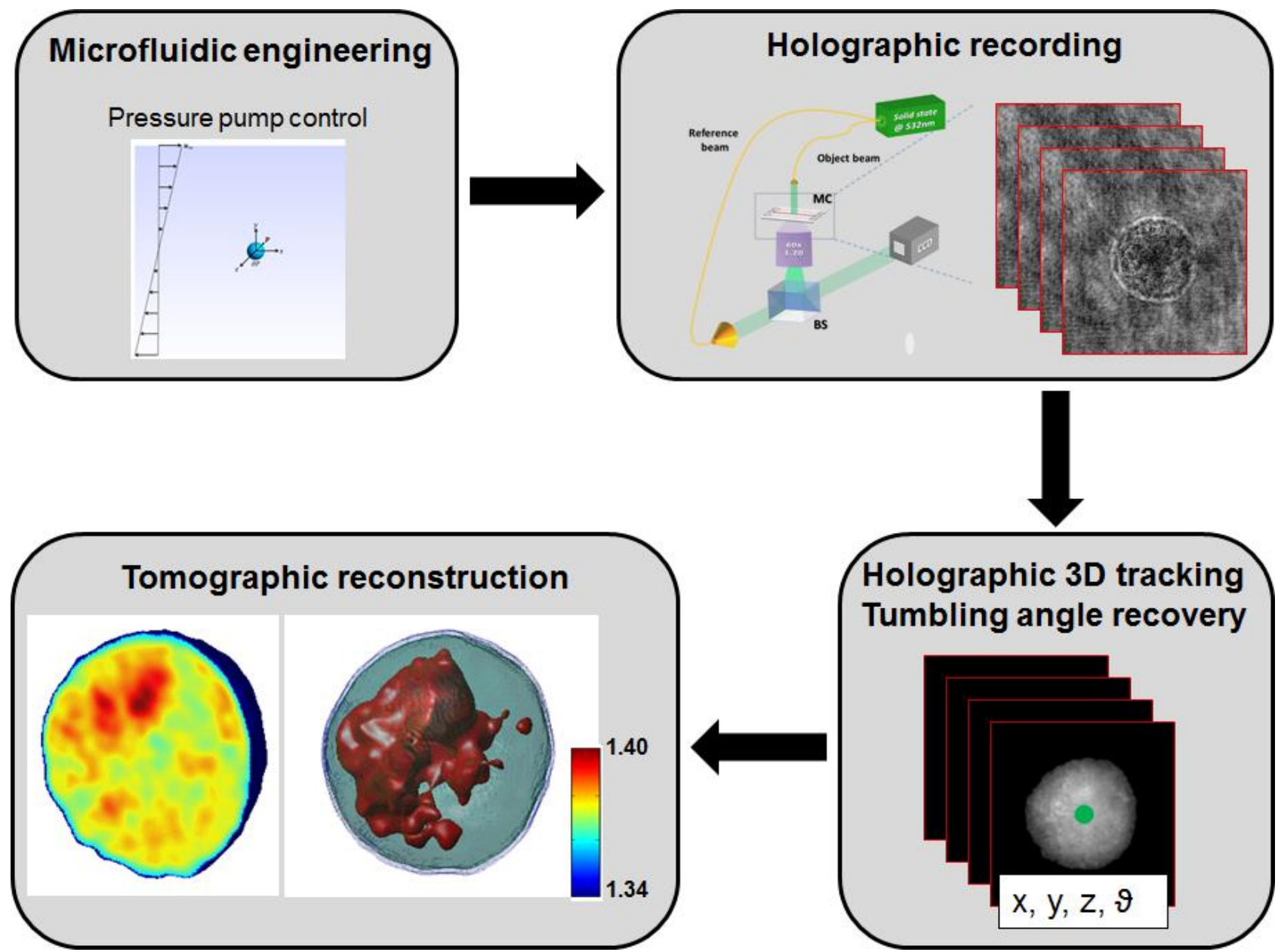

Fig. 1. Continuous TFC by microfluidic engineering.

\section{References}

1. P. Ferraro, A. Wax, Z. Zalevsky, Coherent Light Microscopy (Series in Surface Sciences, Springer 46 2011).

2. Y.K. Park, C. Depeursinge, G. Popescu, Nat Photon 12, 578 - 589 (2018).

3. F. Merola, P. Memmolo, L. Miccio, V. Bianco, M. Paturzo, P. Ferraro, Proc. IEEE 103(2), 192 - 204 (2015).

4. B. Kemper, I. Bredebusch, W. Domschke, S. Kosmeier, P. Langehanenberg, J. Schnekenburger, G. von Bally, J. Biomed Opt 12, 054009 (2007).

5. P. Memmolo, L. Miccio, F. Merola, O. Gennari, P.A. Netti, P. Ferraro, Cytometry Part A 85A, 1030-1036 (2014).

6. D. Jin, R. Zhou, Z. Yaqoob, P.T.C. So, J. Opt. Soc. Am. B 34, B64-B77 (2017).

7. F. Merola, P. Memmolo, L. Miccio, M. Mugnano, P. Ferraro, Methods 136, 108-115 (2018).
8. W. Choi, C. Fang-Yen, K. Badizadegan, S. Oh, N. Lue, R.R. Dasari, M.S. Feld, Nat Methods 4, 717-719 (2007)

9. F. Charrière, A. Marian, F. Montfort, J. Kuehn, T. Colomb, E. Cuche, P. Marquet, C. Depeursinge, Opt Lett 31, 178-180 (2006).

10. F. Merola, P. Memmolo, L. Miccio, R. Savoia, M. Mugnano, A. Fontana, G. D'Ippolito, A. Sardo, A. Iolascon, A. Gambale, P. Ferraro, Light Sci. Appl. 6, e16241 (2017).

11. M.M. Villone, P. Memmolo, F. Merola, M. Mugnano, L. Miccio, P.L. Maffettone, P. Ferraro, Lab Chip 18, 126-131 (2018).

12. M. Habaza, B. Gilboa, Y. Roichman, N.T. Shaked, Opt Lett 40, 1881-1884 (2015).

13. M. Padgett, R. Di Leonardo, Lab Chip 11, 1196-1205 (2011).

14. P. Memmolo, L. Miccio, M. Paturzo, G. Di Caprio, G. Coppola, P.A. Netti, P. Ferraro, Adv Opt Photon 7, 713-755 (2015) 